\title{
ON FRACTIONAL INTEGRATION OF GENERALIZED FUNCTIONS ON A HALF-LINE
}

\author{
by B. RUBIN* \\ (Received 25th October 1993)
}

\begin{abstract}
A new approach to fractional integrals of distributions on a half-line is suggested. The results admit an extension to a large class of Mellin convolutions.
\end{abstract}

1991 Mathematics subject classification: 26A33.

\section{Introduction}

For sufficiently good functions $\phi(x), x \in \mathbf{R}_{+}=[0, \infty)$, fractional integrals of the order $\alpha>0$ are defined by

$$
\left(I_{0}^{\alpha} \phi\right)(x)=: \frac{1}{\Gamma(\alpha)} \int_{0}^{x} \frac{\phi(y) d y}{(x-y)^{1-\alpha}}, \quad\left(I_{-}^{\alpha} \phi\right)(x)=: \frac{1}{\Gamma(\alpha)} \int_{x}^{\infty} \frac{\phi(y) d y}{(y-x)^{1-\alpha}} .
$$

A survey of different approaches to the definition of fractional integrals (1) in spaces of generalized functions on $R_{+}$is presented in [1-4]. The spaces considered in [1-4] are not invariant under the action of operators (1). Moreover, in the framework of these spaces it is impossible to give sense to the divergent integrals $I_{-}^{\alpha} \phi, \phi \in L_{p}\left(\mathbf{R}_{+}\right)$for all $\alpha \geqq 1 / p$ because of some singular values of $\alpha$, namely, $\alpha=1 / p, 1 / p+1,1 / p+2, \ldots$ for which the consideration falls.

At the same time there are many problems where it is important to deal with distribution spaces each of which is invariant under fractional integration of any real order. Here one could mention a wide literature related to the theory and applications of embedding theorems for function spaces of a fractional smoothness. As a rule, such spaces are just ranges of some fractional integral operators (interpreted in the distribution sense and acting on $L_{p}$ - or continuous function. In this situation the order $\alpha$ of a fractional integral characterizes the smoothness of elements of such spaces, and it is important to examine embeddings of spaces of functions (or distributions) with different smoothness. In the case of the whole space $\mathbf{R}^{n}$ (in our case $n=1$ ) spaces of test functions and their duals which are invariant under fractional integration of any order were studied by Lizorkin and some other authors (see, e.g., $[5,8]$ and references there).

Our purpose is to examine a possibility of a similar theory on a half-line by using the Mellin transform as a basic natural tool (in the whole space a Fourier transform is used for this purpose). We introduce the space $\Phi_{+}$of test-functions on $\mathbf{R}_{+}$of Lizorkin type

* Supported in part by the Israel National Council for Research and Development and in part by the Edmund Landau Center for Research in Mathematical Analysis sponsored by the Minerva Foundation (Germany). 
(cf. [5]) which is invariant under $I_{0+}^{\alpha}$ for all $\alpha>0$. The action of $I_{-}^{\alpha}$ is defined correctly in the dual space $\Phi_{+}^{\prime}$ (which contains $L_{p}\left(\mathbf{R}_{+}\right)$for all $\left.p \in[1, \infty]\right)$. We also introduce a more general class of spaces by applying the principle of orthogonality to a prescribed collection of power functions. Such spaces are convenient for Mellin convolutions with kernels whose Mellin transform is represented by the quotient of products of $\Gamma$ functions. Concerning examples and applications of such convolutions see $[2,6]$.

It is my pleasure to acknowledge my deep indebtedness to $\mathrm{A}$. C. McBride for the fruitful discussion of the results and for the hospitality during my visit to the University of Strathclyde (Glasgow) in summer 1993. I also wish to express my thanks to the referee for his valuable remarks.

\section{Spaces of test-functions}

Let $S:=S(\mathbf{R})$ be the $S c h w a r t z$ space of infinitely differentiable rapidly decreasing complex-valued functions on the real line $\mathbf{R}, S\left(\mathbf{R}_{+}\right)$be the linear topological space of restrictions of Schwartz functions on the half-line $\mathbf{R}_{+}:=[0, \infty)$ with the topology $\mathscr{T}$ generated by the sequence of norms

$$
\|\omega: S\|_{k}:=\sup _{m \leqq k x>0}(1+x)^{k}\left|\omega^{(m)}(x)\right|, k=0,1,2, \ldots
$$

Consider the following subspaces of $S\left(\mathbf{R}_{+}\right)$:

$$
\begin{gathered}
S_{+}:=\left\{\omega: \omega \in S\left(\mathbf{R}_{+}\right), \lim _{x \rightarrow 0} x^{-k} \omega^{(m)}(x)=0 \forall k, m=0,1,2, \ldots\right\} \\
\Phi_{+}:=\left\{\omega: \omega \in S_{+}, \int_{0}^{\infty} x^{k} \omega(x) d x=0 \forall k=0,1,2, \ldots\right\} .
\end{gathered}
$$

We shall interpret $S_{+}$and $\Phi_{+}$as linear topological spaces with the topology induced by $\mathscr{T}$.

Remark 1.1. Linear spaces (1.2), (1.3) may be endowed with more adequate topologies. For $S_{+}$such topology $\mathscr{T}_{0}$ may be defined by the sequence of norms

$$
\||\omega|\|_{k}:=\sup _{m \leqq k} \sup _{x>0} \Lambda^{k}(x)\left|\omega^{(m)}(x)\right|, \quad \Lambda(x)=\max (1 / x, 1+x) .
$$

Of course, $\mathscr{T}_{0}$ is stronger than $\mathscr{T}$ on $S\left(\mathbf{R}_{+}\right)$, but on $S_{+}$these two topologies are equivalent. This is obvious from the relation

$$
\sup _{x} \frac{1}{x^{k}}\left|\omega^{(m)}(x)\right|=\sup _{x} \frac{1}{x^{k}}\left|\frac{1}{(k-1) !} \int_{0}^{x}(x-y)^{k-1} \omega^{(m+k)}(y) d y\right| \leqq \frac{1}{k !} \sup _{x>0}\left|\omega^{(m+k)}(x)\right|
$$


which implies $\|\omega\|_{k} \leqq\|\omega ; S\|_{2 k}$. (We leave details to the reader.) For the space $\Phi_{+}$ another equivalent topology will be defined below.

Example 1.1. The function

$$
\kappa(x)=\exp \left(-\frac{\log ^{2} x}{4}\right) \sin \left(\frac{\pi}{2} \log x\right)
$$

belongs to $\Phi_{+}$because its Mellin transform has the form

$$
\int_{0}^{\infty} x^{z-1} \kappa(x) d x=2 \int_{0}^{\infty} \exp \left(-\frac{t^{2}}{4}\right) \operatorname{sh}(z t) \sin \frac{\pi t}{2} d t=2 \sqrt{\pi} \exp \left(z^{2}-\frac{\pi}{4}\right) \sin \pi z
$$

(see [7, formula 4.133]).

Let us investigate the behaviour of Mellin transformations

$$
\tilde{\omega}(z):=(M \omega)(z):=\int_{0}^{\infty} \omega(x) x^{z-1} d x, z \in \mathbf{C}
$$

for $\omega \in S_{+}, \Phi_{+}$.

Denote by $\mathscr{M}$ the space of entire functions $f(z)$ satisfying the following growth condition: for any $k=0,1,2 \ldots$ there exists a constant $c_{k}$ such that $|f(z)| \leqq c_{k}(1+|z|)^{-k}$ provided $|\operatorname{Rez}|<k+1$. The topology in $\mathscr{M}$ will be defined by the norms

$$
\|f ; \mathscr{M}\|_{k}:=\sup _{|R e z| \leqq k+1}(1+|z|)^{k}|f(z)|, k=0,1,2, \ldots
$$

Consider a subspace $\mathscr{M}_{0}$ of $\mathscr{M}$ defined by

$$
\mathscr{M}_{0}:=\{f: f \in \mathscr{M}, f(z)=0 \text { for } z=1,2,3, \ldots\}
$$

with its own topology generated by norms

$$
\begin{gathered}
\left\|f: \mathscr{M}_{0}\right\|_{k}:=\sup _{|\operatorname{Rez}| \leqq k+1} M_{k}(z)|f(z)|, k=0,1,2, \ldots, \\
M_{k}(z):=\sup _{m \leqq k} \max \left\{(1+|z|)^{m} ; \prod_{j=1}^{m+1}|z-j|^{-1}\right\} .
\end{gathered}
$$

The product of $|z-j|^{-1}$ in the definition of $M_{k}(z)$ reflects the fact that all functions $f \in \mathscr{M}_{0}$ vanish at the points $z=1,2, \ldots$

Example 1.2. The function $A(z)=e^{z^{2}} \sin (\pi z)$ is an element of $\mathscr{M}_{0}$ (cf. [1.5]). This follows from the equality 


$$
|A(z)|=\frac{1}{\sqrt{2}}(\operatorname{ch}(2 \pi \eta)-\cos (2 \pi \xi))^{1 / 2} \exp \left(\xi^{2}-\eta^{2}\right), z=\xi+i \eta
$$

Theorem 1.1. The Mellin transform is an isomorphism from $S_{+}$onto $\mathscr{M}$ and from $\Phi_{+}$ onto $\mathscr{M}_{0}$.

Proof. Let $f(z)=\tilde{\omega}(z), \omega \in S_{+}, z=\xi+i \eta,|\xi| \leqq k+1$. For $|\eta| \leqq 1$ we have

where

$$
|f(z)| \leqq\left(\int_{0}^{1}+\int_{1}^{\infty}\right)|\omega(x)| x^{\xi-1} d x=: A_{1}+A_{2}
$$

$$
\begin{aligned}
& A_{1}=\left.\frac{1}{(k+1) !} \int_{0}^{1} x^{\xi-1} \mid \int_{0}^{x}(x-y)^{k+1} \omega^{(k+2}\right)(y) d y \mid \\
& \leqq \frac{1}{(k+2) !} \sup _{x>0}\left|\omega^{(k+2)}(x)\right| \int_{0}^{1} x^{k+\xi+1} d x \leqq \frac{1}{(k+2) !}\|\omega ; S\|_{k+2} \\
& A_{2} \leqq \int_{1}^{\infty}|\omega(x)| x^{k} d x \leqq \sup _{x}(1+x)^{k+2}|\omega(x)| \int_{1}^{\infty} \frac{d x}{x^{2}} \leqq\|\omega ; S\|_{k+2}
\end{aligned}
$$

Similarly for $|\eta|>1$ one obtains

where

$$
|f(z)|=\left|\frac{(-1)^{k}}{z(z+1) \cdots(z+k-1)} \int_{0}^{\infty} \omega^{(k)}(x) x^{z+k-1} d x\right| \leqq \frac{c_{k}}{(1+|z|)^{k}}\left(B_{1}+B_{2}\right),
$$

$$
\begin{aligned}
& B_{1}=\int_{0}^{1}\left|\omega^{(k)}(x)\right| x^{\xi+k-1} d x \leqq c_{k} \mid\|\omega ; S\|_{k+2} \\
& B_{2}=\int_{1}^{\infty}\left|\omega^{(k)}(x)\right| x^{\xi+k-1} d x \leqq c_{k}\|\omega ; S\|_{2 k+2}
\end{aligned}
$$

It follows that $f(z) \in \mathscr{M}$ and

$$
\|f ; \mathscr{M}\|_{k} \leqq c_{k}\|\omega ; S\|_{2 k+2}
$$

Conversely, let $f(z) \in \mathscr{M}$. The estimates of the form

$$
|f(z)| \leqq(1+|\eta|)^{-k}|| f ; \mathscr{M} \|_{N}, N \geqq \max (k ;|\xi|-1), k=0,1,2, \ldots,
$$

imply that $f(z)=\tilde{\omega}(z)$, where 


$$
\omega(x)=\frac{1}{2 \pi i} \int_{\xi-i \infty}^{\xi+i \infty} f(z) x^{-z} d z
$$

(The integral on the right hand side does not depend on $\xi$ by virtue of the Cauchy formula.) Let us prove that $\omega \in S_{+}$. We have

$$
\omega^{(m)}(x)=\frac{(-1)^{m} x^{-m}}{2 \pi i} \int_{\xi-i \infty}^{\xi+\infty} f(z)[z(z+1) \cdots(z+m-1)] x^{-z} d z
$$

and

$$
\omega^{(m)}(x)=\frac{(-1)^{m} x^{-\xi-m}}{2 \pi} \int_{-\infty}^{\infty} f(\xi+i \eta)[(\xi+i \eta) \cdots(\xi+i \eta+m-1)] x^{-i \eta} d \eta
$$

The integral (1.9) (and, consequently, the integral in (1.10)) does not depend on $\xi$. Assuming $\xi=-m+l$, for any $l \in \mathbf{Z}$ we obtain

$$
\begin{aligned}
\left|x^{l} \omega^{(m)}(x)\right| & \leqq c_{l, m} \int_{-\infty}^{\infty}|f(-m+l+i \eta)|(1+|\eta|)^{m} d \eta \\
& \leqq c_{l, m}\|f ; \mathscr{M}\|_{k} \int_{-\infty}^{\infty} \frac{d \eta}{(1+|\eta|)^{k-m}} \leqq c_{l, m}\|f ; \mathscr{M}\|_{k}, k=|l|+m+2 .
\end{aligned}
$$

Hence $\omega \in S_{+}$and

$$
\|\omega ; S\|_{m} \leqq c_{m}|| \tilde{\omega} ; \mathscr{M} \|_{2 m+2}
$$

The first assertion is proved.

Further, the continuity of the mapping $M^{-1}: \mathscr{M}_{0} \rightarrow \Phi_{+}$is obvious due to the estimate

$$
\|\tilde{\omega} ; \mathscr{M}\|_{k} \leqq\left\|\tilde{\omega} ; \mathscr{M}_{0}\right\|_{k}
$$

In order to prove the continuity of the mapping $M: \Phi_{+} \rightarrow \mathscr{M}_{0}$ it is sufficient to estimate the expression

$$
g_{m}(z):=|\tilde{\omega}(z)| \prod_{j=1}^{m+1}|z-j|^{-1}, m \leqq k
$$

for $|R e z| \leqq k+1$. One can readily see that the relation $\omega \in \Phi_{+}$implies the equality $I_{0+}^{1} \omega=-I_{-}^{1} \omega \in \Phi_{+}$. Indeed, for $x<1$ and any $k=0,1,2, \ldots$ we have

$$
\left|x^{-k}\left(I_{0}^{1}+\omega\right)(x)\right| \leqq \int_{0}^{x} \frac{|\omega(y)|}{y^{k}} d y \leqq \text { const. }
$$

For $x>1$ we use the equality $I_{0+}^{1} \omega=-I_{-}^{1} \omega$ which leads to 


$$
\left|x^{k}\left(I_{0}^{1}+\omega\right)(x)\right| \leqq \int_{x}^{\infty} y^{k}|\omega(y)| d y \leqq \text { const }
$$

The orthogonality conditions may be easily checked by using integration by parts.

Thus, we have

$$
I_{0+}^{m} \omega=(-1)^{m} I_{-}^{m} \omega \in \Phi_{+} \forall m=1,2, \ldots
$$

By making use of the last formula we have

$$
\tilde{\omega}(z)=(-1)^{m+1} \prod_{j=1}^{m+1}(z-j)\left(u_{m}(z)+v_{m}(z)\right)
$$

where

$$
u_{m}(z)=\int_{0}^{1} x^{z-m-2}\left(I_{0+}^{m+1} \omega\right)(x) d x, v_{m}(z)=(-1)^{m+1} \int_{1}^{\infty} x^{z-m-2}\left(I_{-}^{m+1} \omega\right)(x) d x
$$

Since for $z=\xi+i \eta,|\xi| \leqq k+1$,

$$
\left|u_{m}(z)\right| \leqq \int_{0}^{1} x^{\xi-m-2}\left|\left(I_{0+}^{m+k+3} \omega^{(k+2)}\right)(x)\right| d x \leqq\|\omega ; S\|_{k+2}
$$

and

$$
\left|v_{m}(z)\right| \leqq \int_{1}^{\infty} x^{\xi-m-2} d x \int_{x}^{\infty} y^{m}|\omega(y)| d y \leqq \int_{1}^{\infty} \frac{d x}{x^{2}} \int_{x}^{\infty} y^{k+1}|\omega(y)| d y \leqq\|\omega ; S\|_{k+3},
$$

then

$$
\left|g_{m}(z)\right| \leqq 2\|\omega ; S\|_{k+3}
$$

Now the required continuity follows from the simple estimate

which gives

$$
\left\|\tilde{\omega} ; \mathscr{M}_{0}\right\|_{k} \leqq \max \left\{\|\tilde{\omega} ; \mathscr{M}\|_{k} ; \sup _{|R e z| \leqq k+1} \sup _{m \leqq k}\left|g_{m}(z)\right|\right\}
$$

$$
\left\|\tilde{\omega} ; \mathscr{M}_{\mathrm{o}}\right\|_{k} \leqq c_{k}\|\omega ; S\|_{2 k+3}
$$

according to (1.8), (1.15).

Corollary 1.1. The space $S_{+}$(the space $\Phi_{+}$) is an algebra (with respect to the Mellin convolution) which is isomorphic to the multiplicative algebra $\mathscr{M}\left(\mathscr{M}_{0}\right)$. The space $\Phi_{+}$is an ideal in the algebra $S_{+}$.

Remark 1.2. Estimates (1.12), (1.13), (1.16) show that topologies defined by norms (1.6) and (1.7) are equivalent on $\mathscr{M}_{0}$. By Theorem 1.1, the topology on $\mathscr{M}_{0}$ defined by 
(1.7) induces a natural topology on $\Phi_{+}$which is equivalent (on $\left.\Phi_{+} !\right)$to that defined by (1.1). A similar situation occurs for the Lizorkin spaces $\Phi\left(\mathbf{R}^{n}\right)$ (see $\left.[5,8]\right)$, the topology of which is defined by using the Fourier transform.

\section{Fractional integrals in the spaces of test functions and in the spaces of distributions}

The space $\Phi_{+}$is invariant under $I_{0+}^{\alpha}, I_{-}^{\alpha}$ for integral $\alpha$ (cf. (1.14)). In the general case we have the following:

Theorem 2.1. The operator $I_{0+}^{\alpha}$, Re $\alpha>0$, is an automorphism of the space $\Phi_{+}$. There is no subspace $X \neq\{0\}$ of the space $S_{+}$such that $X$ is invariant under $I_{-}^{\alpha}$ for all $\alpha>0$.

Proof. Let us verify the first statement. For $\omega \in \Phi_{+}$we have

$$
\left(I_{0}^{\alpha}+\omega\right)^{\sim}(z)=\frac{\Gamma(1-\alpha-z)}{\Gamma(1-z)} \tilde{\omega}(z+\alpha):=f_{\alpha}(z), \operatorname{Re} z<1-\operatorname{Re} \alpha,
$$

where $f_{\alpha}(z)$ is an entire function of $z$ for all $\alpha \in \mathbf{C}$ with the zeroes $z=1,2, \ldots$ Let us prove that $f_{\alpha}(z) \in \mathscr{M}_{0}$. To do this we estimate the norm (1.7). Assume $\alpha=\gamma+i \delta, z=\xi+i \eta$, $|\xi| \leqq k+1$. Then $|\operatorname{Re}(z+\alpha)| \leqq s+1, s=k+2+[|\gamma|]([|\gamma|]$ stands for the entire part of $|\gamma|)$, and hence

$$
|\tilde{\omega}(z+\alpha)| \leqq c_{k}(1+|z+\alpha|)^{-s}\left\|\tilde{\omega} ; \mathscr{M}_{\mathrm{o}}\right\|_{s}
$$

According to the estimate

$$
|\Gamma(1-\alpha-z) / \Gamma(1-z)| \leqq c_{k}|z|^{-\gamma}
$$

which holds for $|\xi| \leqq k+1,|\eta|>1+\delta$, for such $\xi, \eta$ we have

$$
\left|f_{\alpha}(z)\right| \leqq c_{k}(1+|z|)^{-k}|| \tilde{\omega} ; \mathscr{M}_{0} \|_{s}
$$

with some constant $c_{k}$ which may depend also on $\alpha$. If $|\xi| \leqq k+1,|\eta| \leqq 1+\delta$, then

$$
\begin{aligned}
\left|f_{\alpha}(z)\right|= & \prod_{j=1}^{k+1}|z-j|\left|\frac{\Gamma(1-\alpha-z)^{k+1}}{\Gamma(1-z)} \prod_{j=1}(z-j)^{-1} \prod_{j=1}^{s+1}(z+\alpha-j)\right| \\
& \times\left|\tilde{\omega}(z+\alpha) \prod_{j=1}^{s+1}(z+\alpha-j)^{-1}\right| \leqq c_{k}|| \tilde{\omega} ; \mathscr{M}_{0} \|_{s} \prod_{j=1}^{k+1}|z-j| .
\end{aligned}
$$

Hence $\left\|f_{a} ; \mathscr{M}_{0}\right\|_{k} \leqq c_{k}\left\|\tilde{\omega} ; \mathscr{M}_{0}\right\|_{s}$ which implies the continuity of the mapping $\mathscr{M}_{0} \ni \tilde{\omega} \rightarrow$ $f_{a} \in \mathscr{M}_{0} \forall \alpha \in \mathbf{C}$ and yields the desired result by virtue of Theorem 1. 
In order to prove the second statement we assume that there exists a subspace $X \subset S_{+}$ which is invariant under $I_{-}^{\alpha}$ for all $\alpha>0$. Then the equality

$$
\left(I_{-}^{\alpha} \omega\right)^{\sim}(z)=\frac{\Gamma(z)}{\Gamma(z+\alpha)} \tilde{\omega}(z+\alpha), \operatorname{Rez}>0, \omega \in X
$$

yields the relation $\tilde{\omega}(z+\alpha) \Gamma(z) / \Gamma(z+\alpha) \in \mathscr{M} \forall \alpha>0$. The latter is possible only for $\omega(x) \equiv 0$.

The equality (2.2) leads to a natural idea, namely, to consider the action of $I_{-}^{\alpha}$ on functions from the space

$$
\Phi_{\alpha}:=\left\{\omega: \omega \in S_{+} ; \int_{0}^{\infty} \omega(x) x^{\alpha-k} d x=0, k=1,2, \ldots\right\}
$$

with the topology of $S_{+}$. Then Theorem 1.1 yields the following assertion.

Theorem 2.2 The operator $I_{-}^{\alpha}$, Re $\alpha>0$, is an isomorphism from $\Phi_{\alpha}$ onto $\Phi_{-\alpha}$.

Let us pass to the consideration of generalized functions. Denote by $\Phi_{+}^{\prime}, \Phi_{\alpha}^{\prime}$ the duals of $\Phi_{+}, \Phi_{\alpha}$ respectively. The convergence in $\Phi_{+}^{\prime}, \Phi_{\alpha}^{\prime}$ is defined as a weak convergence of functionals. We define

$$
\begin{aligned}
& \left(I_{-}^{\alpha} f, \omega\right)=:\left(f, I_{0+}^{\alpha} \omega\right), \omega \in \Phi_{+}, f \in \Phi_{+}^{\prime}, \\
& \left(I_{0+}^{\alpha} f, \omega\right)=:\left(f, I_{-}^{\alpha} \omega\right), \omega \in \Phi_{\alpha}, f \in \Phi_{-\alpha}^{\prime} .
\end{aligned}
$$

The following statement is obvious due to Theorems 2.1, 2.2.

Theorem 2.3. For all $R e \alpha>0$ the operators $I_{-}^{\alpha}: \Phi_{+}^{\prime} \rightarrow \Phi_{+}^{\prime}, I_{0+}^{\alpha}: \Phi_{-\alpha}^{\prime} \rightarrow \Phi_{\alpha}^{\prime}$ are isomorphisms.

Remark 2.1. When proving isomorphism properties of fractional integrals (see Theorems $2.1,2.2,2.3$ ) we actually did not use any analytic form of corresponding fractional derivatives. Necessary information which covers all complex $\alpha$ was presented in terms of the Mellin transform, isomorphism properties of which are established in Theorem 1.1. In the context of the paper fractional derivatives are well defined by analytical continuation in $(2.1),(2.2)$ for $\omega \in \Phi_{+}$and $\omega \in \Phi_{-\alpha}$ respectively.

\section{Some generalizations}

Let $r=\left\{a_{j}, \alpha_{j}\right\}_{j=1}^{m}$ be a sequence of pairs of numbers $a_{j} \in \mathbf{C}$ and $\alpha_{j}(\neq 0) \in \mathbf{R}$. Introduce the space 


$$
\Phi_{r}=: \Phi_{r}\left(\mathbf{R}_{+}\right)=:\left\{\omega: \omega \in S_{+} ; \int_{0}^{\infty} \omega(x) x^{a_{j}+a_{j} k-1} d x=0, j=1, \ldots, m, k=0,1,2, \ldots\right\}
$$

with the standard topology of the space $S_{+}$. As an example of a function from $\Phi_{r}$ one can take

$$
\kappa_{r}(x)=\left(\kappa_{1} * \cdots * \kappa_{m}\right)(x), \kappa_{j}(x)=x^{\alpha_{j}-a_{j}} \kappa\left(x^{\alpha_{j}}\right)
$$

$\kappa(x)$ being the function (1.4), “*” standing for the Mellin convolution. The space $\Phi_{r}$ is complete and is an ideal in the convolution algebra $S_{+}$.

Consider the operator $K$ which is defined on functions $\omega(x), x \in \mathbf{R}_{+}$by the relation

$$
(K \omega)^{-}(z)=\mathscr{K}(z) \tilde{\omega}(\delta+\lambda z), \mathscr{K}(z)=\prod_{j=1}^{A} \Gamma\left(a_{j}+\alpha_{j} z\right) / \prod_{j=1}^{B} \Gamma\left(b_{j}+\beta_{j} z\right)
$$

where $a_{j}, b_{j}, \delta \in \mathbf{C}$ and $\alpha_{j}, \beta_{j} \lambda \in \mathbf{R}$ are some numbers, $\lambda \neq 0$. A large number of integrodifferential operators on $\mathbf{R}_{+}$can be represented in the form $(3.1)$ (see $[2,6]$ ).

Theorem 3.1. Let $A=B:=m, \alpha_{j}=\beta_{j}, j=1, \ldots, m$,

$$
r=\left\{\delta-\frac{\lambda a_{j}}{\alpha_{j}},-\frac{\lambda}{\alpha_{j}}\right\}_{j=1}^{m}, \eta=\left\{-\frac{b j}{\alpha_{j}},-\frac{1}{\alpha_{j}}\right\}_{j=1}^{m}
$$

Then the operator $K$ defined by (3.1) is an isomorphism from $\Phi_{r}$ onto $\Phi_{\eta}$.

The proof of this theorem is similar to the proof of Theorem 2.1. Note, that if $A \neq B$ or $\alpha_{j} \neq \beta_{j}$ for some $j$, then $K$ cannot be an isomorphism in the spaces under consideration, because in this case multiplication (or division) by $\mathscr{K}(z)$ does not leave the space $\mathscr{M}$ invariant.

Corollary 3.1. Assume that under conditions of Theorem 3.1 the following relation hold:

$$
\lambda=1, \delta=\left(a_{j}-b_{j}\right) / \alpha_{j}, j=1,2, \ldots, m \text {. }
$$

Then $K$ is an automorphism of the space $\Phi_{r}$.

In conclusion we note that Theorem 3.1 enables us to define the action of the adjoint of the operator (3.1) in the corresponding spaces $\Phi_{r}^{\prime}$ of distributions.

\section{REFERENCES}

1. A. C. McBride, Fractional calculus and integral transforms of generalized functions (Research Notes in Math. 31, Pitman, London, 1979). 
2. A. C. McBride, A Mellin transform approach to fractional calculus on $(0, \infty)$, in Fractional Calculus (Research Notes in Math. 138, Pitman, London, 1985), 99-139.

3. A. ERDÉLYI, Fractional integrals of generalized functions, Lecture Notes in Math. 457 (1975), 151-170.

4. Y. A. Brychkov, A. P. Prudnikov, Integral transforms of generalized functions (Moscow, 1977 (in Russian)).

5. P. I. Lizorkin, Generalized Liouville differentiation and the method of multipliers in the theory of imbeddings of classes of differentiable functions, Proc. Steklov. Inst. Math. 105 (1969), 105-202.

6. Y. A. Brychkov, H.-J. Glaeske and O. I. Marichev, The factorization of integral transforms of the convolution type, Itogi Nauki i Tekhniki., VINITI, Math. Anal. 21 (1983), 3-41.

7. I. S. Gradshteyn and I. M. RyzhiK, Table of Integrals, Series and Products Academic Press 1980.

8. S. G. Sамко, Hypersingular integrals and their applications (Rostov-on-Don, 1984 (in Russian).

Department of Mathematics

The Hebrew University of Jerusalem

Givat Ram 91904

JERUSALEM

ISRAEL

Email: boris@humus.huji.ac.il 